\title{
Entanglement-assisted quantum feedback control
}

\author{
Naoki Yamamoto $^{1}$ - Tomoaki Mikami ${ }^{1}$
}

Received: 17 August 2016 / Accepted: 27 May 2017 / Published online: 7 June 2017

C The Author(s) 2017. This article is an open access publication

\begin{abstract}
The main advantage of quantum metrology relies on the effective use of entanglement, which indeed allows us to achieve strictly better estimation performance over the standard quantum limit. In this paper, we propose an analogous method utilizing entanglement for the purpose of feedback control. The system considered is a general linear dynamical quantum system, where the control goal can be systematically formulated as a linear quadratic Gaussian control problem based on the quantum Kalman filtering method; in this setting, an entangled input probe field is effectively used to reduce the estimation error and accordingly the control cost function. In particular, we show that, in the problem of cooling an opto-mechanical oscillator, the entanglement-assisted feedback control can lower the stationary occupation number of the oscillator below the limit attainable by the controller with a coherent probe field and furthermore beats the controller with an optimized squeezed probe field.
\end{abstract}

Keywords Quantum metrology · Entanglement · Feedback control · Continuous measurement $\cdot$ Opto-mechanics $\cdot$ Feedback cooling

\section{Introduction}

Entanglement is a special notion that had been considered as a "spooky" correlation [1]. However, over recent decades it has gained a positive impression mainly thanks to its

\footnotetext{
This work was supported in part by JSPS Grant-in-Aid No. 15K06151 and JST PRESTO No. JPMJPR166A. The authors acknowledge helpful discussions with M. R. Hush, A. R. R. Carvalho, and S. S. Szigeti.

$凶 \quad$ Naoki Yamamoto yamamoto@appi.keio.ac.jp

1 Department of Applied Physics and Physico-Informatics, Keio University, Hiyoshi 3-14-1, Kohoku, Yokohama 223-8522, Japan
} 
(a)

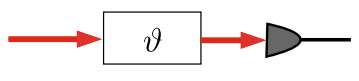

(b)

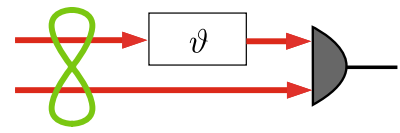

Fig. 1 a Standard setup for estimating an unknown parameter $\vartheta$. b Quantum metrology setup using an entangled input

central role in quantum information science [2,3]. A particularly important application of entanglement in our context is the quantum metrology [4,5]. The basic configuration is depicted in Fig. 1. The goal is to estimate an unknown parameter $\vartheta$ of the system. A standard estimation method is first to send a known input state and then measure the output state containing the information about $\vartheta$ (Fig. 1a); in this case, the estimation error has a strict lower bound called the standard quantum limit ( $S Q L$ ) with respect to the input energy. In the quantum metrology schematic depicted in Fig. 1b, on the other hand, an entangled state is chosen as an input so that one portion passes through the system while the other portion does not; then by measuring the combined output, we obtain more information about $\vartheta$ than the standard case (Fig. 1a) and thus can beat the SQL in the estimation error. This schematic has been experimentally demonstrated in several settings, e.g., [6-9]. Note that the entanglement-assisted method is not a unique approach for beating the SQL; particularly in the case where $\vartheta$ is the parameter of a force applied to a mechanical oscillator (e.g., a gravitational wave force), several alternative estimation schemes beating the SQL have been developed, such as the squeezed probe scheme [10-13] and the variational measurement technique [14] for back-action evasion.

What we learn from the theory of quantum metrology is the fact that, in a broad sense, a quantum estimator could have better performance if assisted by entanglement. Therefore, it is a reasonable idea to employ an entanglement-assisted estimation strategy for the measurement-based quantum feedback control $[15,16]$, which is now well established based on the quantum filtering theory [17,18]. Actually, in a similar configuration depicted in Fig. 1b, it is expected that the quantum filter (i.e., the best continuous-time estimator) brings us more information, and as consequence we will have chance to construct a better controller than in the standard case without entanglement. The idea of entanglement-assisted feedback control is briefly mentioned in [19], but there has been no quantitative analysis of this control strategy. That is, we are interested in the following questions; (i) How much does the entanglementassisted strategy improve the control performance in a realistic setup? (ii) In what situation is the entanglement-assisted feedback control really beneficial? Note that the answers to these questions are non-trivial, because, for a realistic noisy system, the entanglement-assisted feedback control will not always outperform the standard one without entanglement, due to the fragile nature of entangled states.

In this paper, we consider the setup illustrated in Fig. 2. The system to be controlled is a general linear quantum system such as an optical amplifier and an opto-mechanical oscillator [20-25]. The probe input is given by an optical entangled state generated by combining a squeezed field and a coherent field [26] at a beam splitter; one portion of 


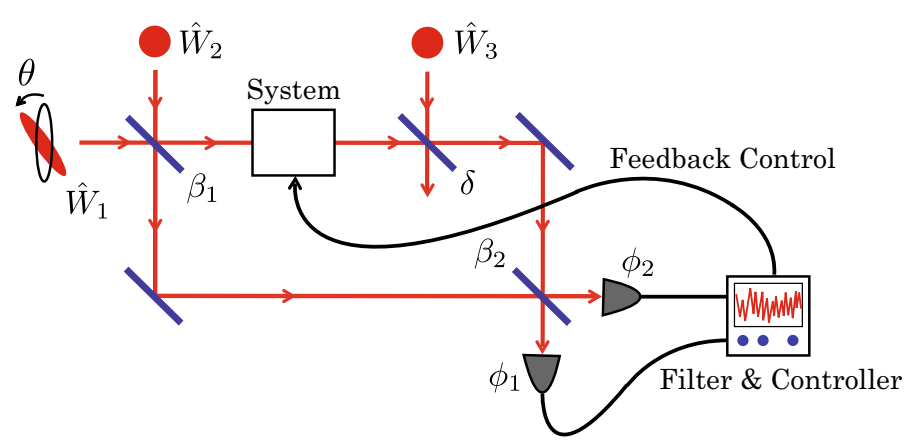

Fig. 2 General feedback control configuration via an entangled input field. $\hat{W}_{1}$ and $\hat{W}_{2}$ are field annihilation operators representing a squeezed state with phase $\theta$ and a coherent (or vacuum) state, respectively. $\hat{W}_{3}$ represents a vacuum field. $\beta_{1}^{2}, \beta_{2}^{2}$, and $\delta^{2}$ denote the reflectivity of the beam splitters; in particular $\delta$ corresponds to an optical loss of the system's output field. $\phi_{1}$ and $\phi_{2}$ are the phases of Homodyne detectors

this entangled field couples to the system while the other portion does not, as in the scheme shown in Fig. 1b; and then the combined output field is continuously measured by Homodyne detectors. Finally, based on the measurement signal, we construct the quantum filter and then apply a feedback control to the system. The point of this setting is that the system state is always Gaussian, and as a result the quantum filter is simplified to the quantum Kalman filter [27], which enables us to compute the exact real-time estimate of the system variables. Furthermore, in this paper, we consider the quantum linear quadratic Gaussian $(L Q G)$ optimal control problem, meaning that the control goal is to minimize a quadratic-type cost function. Fortunately, again thanks to the Gaussianity of the system state, this problem can be analytically solved by almost the same way as in the classical case [28,29]. An important fact in this formulation is that a strict lower bound of the cost, which is ideally achievable by employing the so-called cheap control [30-32], is represented by a function of only the estimation error. Therefore, this ultimate limit of the LQG control cost has the meaning of SQL, when the input is given by a coherent or a vacuum field.

In the above-described framework, first, this paper proves that, thanks to the entangled probe input, the filter certainly gains additional information which may improve the control performance. Next, we study a feedback cooling problem of an optomechanical oscillator [33-38] and provide answers to the above-posed questions by conducting detailed numerical simulations. In particular, it is shown that, by carefully choosing the system parameters $\left(\theta, \beta_{1}, \beta_{2}, \phi_{1}, \phi_{2}\right.$; see Fig. 2), the entanglementassisted feedback control can lower the stationary occupation number of the oscillator below the SQL in the sense of cheap control mentioned above, and moreover, it outperforms the control with an optimized squeezed probe field [13].

Finally, we note that the scheme presented in this paper differs from that studied in [36], which considers the use of system's internal entanglement to enhance cooling for an opto-mechanical system.

Notation: $\Re$ and $\Im$ denote the real and imaginary parts, respectively. $I_{n}: n \times n$ identity matrix. $O_{n}: n \times n$ zero matrix. $0_{n \times m}: n \times m$ zero matrix. 


\section{Quantum Kalman filtering, LQG control, and cheap control}

We here review the general theory of quantum Kalman filtering, LQG optimal control, and the cheap control.

\subsection{Quantum linear systems}

In this paper, we consider a linear quantum system, whose general form is described as follows (see $[15,16,21,24]$ for more details). The system variables are collected in a vector of operators $\hat{x}:=\left[\hat{q}_{1}, \hat{p}_{1}, \ldots, \hat{q}_{n}, \hat{p}_{n}\right]^{\top}$, where $\hat{q}_{i}$ and $\hat{p}_{i}$ are position and momentum operators. They satisfy the canonical commutation relation $\hat{q}_{i} \hat{p}_{j}-\hat{p}_{j} \hat{q}_{i}=$ $i \delta_{i j}($ we set $\hbar=1)$, which is summarized as

$$
\hat{x} \hat{x}^{\top}-\left(\hat{x} \hat{x}^{\top}\right)^{\top}=i \Sigma_{n}
$$

where $\Sigma_{n}$ is the following $2 n \times 2 n$ block diagonal matrix:

$$
\Sigma_{n}=\operatorname{diag}\{\sigma, \ldots, \sigma\}, \quad \sigma=\left[\begin{array}{cc}
0 & 1 \\
-1 & 0
\end{array}\right]
$$

The system variables are governed by the linear dynamics

$$
d \hat{x}_{t}=A \hat{x}_{t} d t+F u_{t} d t+B d \hat{W}_{t}
$$

Note that, in order to preserve Eq. (1) for all $t$, the matrix $A$ must be of the following form:

$$
A=\Sigma_{n}\left(G+\Sigma_{n}^{\top} B \Sigma_{m} B^{\top} \Sigma_{n} / 2\right),
$$

where $G$ is a $2 n \times 2 n$ real symmetric matrix determining the system Hamiltonian by $\hat{H}=\hat{x}^{\top} G \hat{x} / 2$. Also $B$ is a $2 n \times 2 m$ real matrix determined from the systemfield coupling. $F$ is a real matrix, and $u_{t}$ is the vector of classical (i.e., non-quantum) signal representing the control input. The system couples with $m$ probe or environment bosonic fields, with vector of noise operators $\hat{W}_{t}:=\left[\hat{Q}_{1}, \hat{P}_{1}, \ldots, \hat{Q}_{m}, \hat{P}_{m}\right]^{\top}$. This satisfies the quantum Ito rule $d \hat{W}_{t} d \hat{W}_{t}^{\top}=\Theta d t$, with zero mean: $\left\langle d \hat{W}_{t}\right\rangle=0$. The correlation matrix $\Theta$ is $2 m \times 2 m$ block diagonal Hermitian, and their $j$ th block matrix (i.e., the correlation matrix of $\hat{Q}_{j}$ and $\hat{P}_{j}$ ) is in general written as

$$
\Theta_{j}=\left[\begin{array}{cc}
N_{j}+\Re\left(M_{j}\right)+1 / 2 & \Im\left(M_{j}\right)+i / 2 \\
\Im\left(M_{j}\right)-i / 2 & N_{j}-\Re\left(M_{j}\right)+1 / 2
\end{array}\right] .
$$

The parameters $N_{j} \in \mathbb{R}$ and $M_{j} \in \mathbb{C}$ satisfy $N_{j}\left(N_{j}+1\right) \geq\left|M_{j}\right|^{2}$. Note that $N_{j}$ represents the average excitation number of the probe quanta, and $M_{j}$ is related to squeezing of the field; if $N_{j}\left(N_{j}+1\right)=\left|M_{j}\right|^{2}$ is satisfied, the probe field state is a 
pure squeezed state, ${ }^{1}$ while if $M_{j}=0$ it is not squeezed. Also note that $d \hat{Q}_{j} d \hat{P}_{j}-$ $d \hat{P}_{j} d \hat{Q}_{j}=i d t$.

For this system, we perform a (joint) Homodyne measurement on $\ell(\leq m)$ output probe fields, which generates the classical measurement signal

$$
d y_{t}=C \hat{x}_{t} d t+D d \hat{W}_{t}
$$

Note that, due to the unitarity of the system-field coupling, the $\ell \times 2 n$ real matrix $C$ and the $\ell \times 2 m$ real matrix $D$ satisfy the following specific structure:

$$
C=D \Sigma_{m} B^{\top} \Sigma_{n}, \quad D \Sigma_{m} D^{\top}=0
$$

In this paper, we assume that $A$ is Hurwitz, meaning that the real parts of all the eigenvalues of $A$ are negative; hence, in this case the mean of the system variables, $\left\langle\hat{x}_{t}\right\rangle$, which obeys the dynamics $d\left\langle\hat{x}_{t}\right\rangle / d t=A\left\langle\hat{x}_{t}\right\rangle$, converges to zero in the long time limit, i.e., $\left\langle\hat{x}_{t}\right\rangle \rightarrow 0$. Note that the opto-mechanical system studied in Sect. 4 is Hurwitz.

\subsection{Quantum Kalman filter}

Let us consider the situation where we want to perform a real-time estimate of the system variable $\hat{x}_{t}$ based on the measurement signal $y_{t}$. The solution is provided by the quantum filtering theory; that is, we can rigorously define the quantum conditional expectation $\pi\left(\hat{x}_{t}\right):=\mathbb{E}\left(\hat{x}_{t} \mid \mathcal{Y}_{t}\right)$, where $\mathcal{Y}_{t}$ is the $\sigma$-algebra composed of the measurement signal $\left\{y_{s} \mid 0 \leq s \leq t\right\}$. In fact the classical random variable $\pi\left(\hat{x}_{t}\right)$ is the least mean squared estimate of $\hat{x}_{t}$. The recursive equation updating $\pi\left(\hat{x}_{t}\right)$ is given by the quantum Kalman filter [27-29]:

$$
\begin{aligned}
d \pi\left(\hat{x}_{t}\right) & =A \pi\left(\hat{x}_{t}\right) d t+F u_{t} d t+K_{t}\left(d y_{t}-C \pi\left(\hat{x}_{t}\right) d t\right), \\
K_{t} & =\left(V_{t} C^{\top}+B \Re(\Theta) D^{\top}\right)\left(D \Re(\Theta) D^{\top}\right)^{-1},
\end{aligned}
$$

where the initial condition is $\pi\left(\hat{x}_{0}\right)=\left\langle\hat{x}_{0}\right\rangle$ with $\langle\bullet\rangle$ the unconditional expectation. $V_{t}$ is the estimation error covariance matrix defined as

$$
V_{t}:=\left\langle\Delta \hat{x}_{t} \Delta \hat{x}_{t}^{\top}+\left(\Delta \hat{x}_{t} \Delta \hat{x}_{t}^{\top}\right)^{\top}\right\rangle / 2, \Delta \hat{x}_{t}:=\hat{x}_{t}-\pi\left(\hat{x}_{t}\right)
$$

which evolves in time according to the following Riccati differential equation:

$$
\dot{V}_{t}=A V_{t}+V_{t} A^{\top}+B \Re(\Theta) B^{\top}-K_{t} D \Re(\Theta) D^{\top} K_{t}^{\top} .
$$

\footnotetext{
1 The field annihilation operator $\hat{A}_{1}=\left(\hat{Q}_{1}+i \hat{P}_{1}\right) / \sqrt{2}$ for a pure squeeze state is modeled by the Bogoliubov transformation $\hat{A}_{1}=\hat{A}_{1}^{(0)} \cosh (r / 2)-\hat{A}_{1}^{(0)} e^{i \theta / 2} \sinh (r / 2)$, where $\hat{A}_{1}^{(0)}$ is the vacuum field operator. The corresponding parameters $N_{1}$ and $M_{1}$ are obtained from the definition $d \hat{A}_{1} d \hat{A}_{1}^{\dagger}=$ $\left(N_{1}+1\right) d t, d \hat{A}_{1}^{\dagger} d \hat{A}_{1}=N_{1} d t, d A_{1}^{2}=M_{1} d t$ and $d A_{1}^{\dagger 2}=M_{1}^{*} d t$, which lead to $N_{1}=\sinh ^{2}(r / 2)$ and $M_{1}=-e^{i \theta / 2} \sinh (r / 2) \cosh (r / 2)$. Hence, certainly $N_{1}\left(N_{1}+1\right)=\left|M_{1}\right|^{2}$ is satisfied.
} 
Under the assumption $A$ being Hurwitz, ${ }^{2}$ this equation has a unique steady solution $V_{\infty}>0$.

\subsection{Quantum LQG control}

In the infinite horizon quantum LQG control problem, we consider the following cost function:

$$
J[u]=\lim _{T \rightarrow \infty} \frac{1}{T}\left\langle\int_{0}^{T}\left(\hat{x}_{t}^{\top} Q \hat{x}_{t}+u_{t}^{\top} R u_{t}\right) \mathrm{d} t\right\rangle,
$$

where $Q \geq 0$ and $R>0$ are real weighting matrices. The goal is to design the feedback control law $u_{t}$ as a function of $y_{t}$ that minimizes the cost (9) under condition (2), i.e., $u_{t}^{*}=\arg \min _{u} J[u]$. The point is that, due to the tower property $\left\langle\hat{x}_{t}\right\rangle=\left\langle\pi\left(\hat{x}_{t}\right)\right\rangle=$ $\left\langle\mathbb{E}\left(\hat{x}_{t} \mid \mathcal{Y}_{t}\right)\right\rangle$, the cost can be represented in terms of only the filter variable as follows. That is, due to the relation $\mathbb{E}\left(\hat{x}_{t} \hat{x}_{t}^{\top} \mid \mathcal{Y}_{t}\right)=V_{t}+i \Sigma_{n} / 2+\pi\left(\hat{x}_{t}\right) \pi\left(\hat{x}_{t}\right)^{\top}$, we have

$$
\begin{aligned}
& \left\langle\int_{0}^{T}\left(\hat{x}_{t}^{\top} Q \hat{x}_{t}+u_{t}^{\top} R u_{t}\right) \mathrm{d} t\right\rangle=\left\langle\int_{0}^{T}\left(\operatorname{Tr}\left[Q \mathbb{E}\left(\hat{x}_{t} \hat{x}_{t}^{\top} \mid \mathcal{Y}_{t}\right)\right]+u_{t}^{\top} R u_{t}\right) \mathrm{d} t\right\rangle \\
& =\left\langle\int_{0}^{T}\left(\pi\left(\hat{x}_{t}\right)^{\top} Q \pi\left(\hat{x}_{t}\right)+u_{t}^{\top} R u_{t}\right) \mathrm{d} t\right\rangle+\int_{0}^{T} \operatorname{Tr}\left[Q\left(V_{t}+\frac{i}{2} \Sigma_{n}\right)\right] \mathrm{d} t,
\end{aligned}
$$

where we have used the fact that $V_{t}$ obeys the deterministic time evolution (8). Hence, this equation leads to

$$
J[u]=\lim _{T \rightarrow \infty} \frac{1}{T}\left\langle\int_{0}^{T}\left(\pi\left(\hat{x}_{t}\right)^{\top} Q \pi\left(\hat{x}_{t}\right)+u_{t}^{\top} R u_{t}\right) \mathrm{d} t\right\rangle+\operatorname{Tr}\left(Q V_{\infty}\right) .
$$

Note that the second term is constant. As a result, our problem is to find $u_{t}$ minimizing the first term of Eq. (10) under condition (7). This is exactly the classical LQG control problem and can be analytically solved as follows (see [40] or "Appendix A"); the optimal control input is given by

$$
u_{t}^{*}=-R^{-1} F^{\top} P_{t} \pi\left(\hat{x}_{t}\right)
$$

where $P_{t}$ is the solution of the following Riccati equation:

$$
\dot{P}_{t}+P_{t} A+A^{\top} P_{t}-P_{t} F R^{-1} F^{\top} P_{t}+Q=0 .
$$

Likewise the case of Eq. (8), because $A$ is Hurwitz, ${ }^{3}$ this equation has a unique steady solution $P_{\infty} \geq 0$. The minimum value of the cost, which is reached by the optimal

\footnotetext{
${ }^{2}$ Note that the Hurwitz property is a sufficient condition for Eq. (8) to have a unique steady solution. A useful necessary and sufficient condition is that $\left(A^{\top}, D\right)$ is stabilizable and $\left(A^{\top}, B\right)$ is detectable [39].

${ }^{3}$ As mentioned in footnote 2, this condition is stronger than the condition $(A, F)$ being stabilizable and $(A, \sqrt{Q})$ begin detectable, which is a necessary and sufficient condition for Eq. (12) to have a unique steady solution $P_{\infty} \geq 0$. Note that, in this case, $A-F R^{-1} F^{\top} P_{\infty}$ is Hurwitz, meaning that the controlled filter equation is stable.
} 
control (11), is given by

$$
J\left[u^{*}\right]=\operatorname{Tr}\left(K_{\infty} D \Re(\Theta) D^{\top} K_{\infty}^{\top} P_{\infty}\right)+\operatorname{Tr}\left(Q V_{\infty}\right)
$$

Note that $u_{t}^{*}$ is a function of the optimal estimate $\pi\left(\hat{x}_{t}\right)$. Thus, we can design the optimal estimate and control separately; this is called the separation principle as in the classical case [41].

\subsection{Lower bound of the minimum cost: the cheap control}

Let us set $R=\epsilon^{2} I$ for the cost function (9), where $\epsilon>0$ is a positive scalar, and use $P_{\epsilon}$ to denote the solution of the Riccati equation (12). Then, it was proven in [30] that $P_{\epsilon}$ monotonically decreases as $\epsilon$ goes to zero. Moreover, we have that $\lim _{\epsilon \rightarrow 0} P_{\epsilon}=0$, if and only if the system characterized by $(A, F, \bar{Q})$ is minimum phase and right invertible; see "Appendix B" for the definitions of this condition $(\bar{Q}$ is a real matrix satisfying $\left.Q=\bar{Q}^{\top} \bar{Q}\right)$. In this case, the steady solution of the Riccati equation (12) takes the form $P_{\infty}=\epsilon \bar{P}+O\left(\epsilon^{2}\right)$. Then, the minimum cost (13) becomes

$$
J\left[u^{*}\right]=\epsilon \operatorname{Tr}\left(K_{\infty} D \Re(\Theta) D^{\top} K_{\infty}^{\top} \bar{P}\right)+\operatorname{Tr}\left(Q V_{\infty}\right)+O\left(\epsilon^{2}\right),
$$

and the optimal control input (11) is given by $u_{t}^{*}=-\epsilon^{-1} F^{\top} \bar{P} \pi\left(\hat{x}_{t}\right)$ at steady state. Now we consider the situation where the actuator is allowed to have a large control gain (e.g., the case $\epsilon \approx 0$ ); in particular, the control input in the ideal limit $\epsilon \rightarrow+0$, meaning that no penalty is imposed on it, is called the cheap control [30-32]. We then see that this ultimate feedback control perfectly suppresses the fluctuation of the estimated variables $\pi\left(\hat{x}_{t}\right)$, i.e., the first term of Eq. (14), and as a result the total cost is limited only by the optimal estimation error. Therefore, we can take $J^{*}\left[u^{*}\right]=\operatorname{Tr}\left(Q V_{\infty}\right)$ as a fundamental quantity that is reasonably used for evaluating the performance of feedback control, because it cannot be further decreased by any control. In particular, we define the SQL as the value of $J^{*}\left[u^{*}\right]$ when the probe input is given by a coherent or a vacuum field. Finally note that, when $\epsilon \rightarrow+0$, Eq. (9) equals to the stationary energy $\left\langle\hat{x}_{\infty}^{\top} Q \hat{x}_{\infty}\right\rangle$, and this can be ultimately reduced, by the ideal cheap control, to $J^{*}\left[u^{*}\right]=\operatorname{Tr}\left(Q V_{\infty}\right)$.

\section{Configuration of the entanglement-assisted feedback control}

\subsection{Model}

The entanglement-assisted feedback control configuration considered in this paper is depicted in Fig. 2. This model has the following four features.

(i) The system is linear and couples with a single probe field.

(ii) The entangled optical field is produced by combining a fixed squeezed field $\hat{W}_{1}$ and a fixed coherent field $\hat{W}_{2}$, at a beam splitter (BS1). The correlation matrices (4) of these fields are, respectively, given by 


$$
\Theta_{1}=\frac{1}{2}\left[\begin{array}{cc}
\cos \theta & -\sin \theta \\
\sin \theta & \cos \theta
\end{array}\right]\left[\begin{array}{cc}
e^{-r} & i \\
-i & e^{r}
\end{array}\right]\left[\begin{array}{cc}
\cos \theta & \sin \theta \\
-\sin \theta & \cos \theta
\end{array}\right], \quad \Theta_{2}=\frac{1}{2}\left[\begin{array}{cc}
1 & i \\
-i & 1
\end{array}\right],
$$

where $r$ is the squeezing level and $\theta$ represents the phase of the squeezed state in the phase space; see Fig. 2 and footnote in page 5. The reflectivity of BS1 is, for simplicity, set to $\beta_{1}^{2}$ with $0 \leq \beta_{1} \leq 1$. Note that, for all $\beta_{1} \in(0,1)$, the output fields of BS1 are entangled (see "Appendix C"). As shown in the figure, one portion of this entangled field couples with the system, while the other portion does not. The degree of entanglement can be changed by tuning $\beta_{1}$, while maintaining the total amount of energy for producing this entangled field. Hence, we can conduct a fair comparison of the entanglement-assisted control method (the case $0<\beta_{1}<1$ ) and the standard method without entanglement (the case $\beta_{1}=0,1$ ), given the same amount of resources. In particular, note that the SQL corresponds to the case $\beta_{1}=1$.

(iii) We assume that the system's output field is subjected to an optical loss, which can be modeled by introducing a fictitious beam splitter with reflectivity $\delta^{2}$; if $\delta=0$, then there is no optical loss. $\hat{W}_{3}$ denotes the vacuum noise field coming into this fictitious beam splitter, whose correlation matrix is the same as that of $\hat{W}_{2}$, i.e., $\Theta_{3}=$ $\Theta_{2}$. As a consequence, the overall system contains three input fields $\hat{W}_{1}, \hat{W}_{2}$, and $\hat{W}_{3}$, implying that $m=3$ in the system equations (2) and (5). The total correlation matrix is thus given by

$$
\Theta=\operatorname{diag}\left\{\Theta_{1}, \Theta_{2}, \Theta_{3}\right\}
$$

Note again that $\hat{W}_{1}$ and $\hat{W}_{2}$ represent the probe fields, while $\hat{W}_{3}$ denotes the unwanted noise field.

(iv) The system's output field after being subjected to the optical loss meets the other portion of the entangled input field, at the second beam splitter (BS2) with reflectivity $\beta_{2}^{2}$; again for simplicity, we assume $0 \leq \beta_{2} \leq 1$. Then the final output fields are measured by two Homodyne detectors with phase $\phi_{1}$ and $\phi_{2}$, which generate two output signals $y_{1}$ and $y_{2}$. Note that, if $\beta_{2}=0$ or $\beta_{2}=1$, the two optical fields are not combined at BS2 and are measured independently; this type of measurement is called the local measurement. The other case with $0<\beta_{2}<1$ is called the global measurement.

The overall system dynamics realizing the above setup is given as follows. First, we use the fact that, for a general open linear system interacting with a single probe field, the system-field coupling is represented by an operator (the so-called Lindblad operator) of the form $\hat{L}=c^{\top} \hat{x}$ with $c$ the $2 n$-dimensional complex column vector, and this determines the $B$ matrix in Eq. (2) as follows (see e.g., [15,16,24]). That is, by defining the $2 \times 2 n$ real matrix

$$
\bar{C}=\sqrt{2}\left[\begin{array}{c}
\Re(c)^{\top} \\
\Im(c)^{\top}
\end{array}\right]
$$

we can specify the $B$ matrix in the following form:

$$
B=\left[\alpha_{1} \Sigma_{n} \bar{C}^{\top} \Sigma_{1}, \beta_{1} \Sigma_{n} \bar{C}^{\top} \Sigma_{1}, 0_{2 n \times 2}\right],
$$


where $\alpha_{1}=\sqrt{1-\beta_{1}^{2}}$. Then the $A$ matrix is determined by Eq. (3) with $G$ specified by the system Hamiltonian $\hat{H}=\hat{x}^{\top} G \hat{x} / 2$. The $C$ matrix is also specified by Eq. (6) and is now given by

$$
C=D \Sigma_{3} B^{\top} \Sigma_{n}=D\left[\begin{array}{c}
\alpha_{1} \bar{C} \\
\beta_{1} \bar{C} \\
0_{2 \times 2 n}
\end{array}\right]
$$

Here $D$ is a $2 \times 6$ real matrix of the form

$$
D=\left[D_{1}, D_{2}, O_{2}\right] T_{2} T_{L} T_{1}
$$

where

$$
D_{1}=\left[\begin{array}{cc}
\cos \phi_{1} & \sin \phi_{1} \\
0 & 0
\end{array}\right], \quad D_{2}=\left[\begin{array}{cc}
0 & 0 \\
\cos \phi_{2} & \sin \phi_{2}
\end{array}\right]
$$

and

$$
T_{k}=\left[\begin{array}{ccc}
\alpha_{k} I_{2} & \beta_{k} I_{2} & O_{2} \\
-\beta_{k} I_{2} & \alpha_{k} I_{2} & O_{2} \\
O_{2} & O_{2} & I_{2}
\end{array}\right] \quad(k=1,2), \quad T_{L}=\left[\begin{array}{ccc}
\sqrt{1-\delta^{2}} I_{2} & O_{2} & \delta I_{2} \\
O_{2} & I_{2} & O_{2} \\
-\delta I_{2} & O_{2} \sqrt{1-\delta^{2}} I_{2}
\end{array}\right],
$$

with $\alpha_{2}=\sqrt{1-\beta_{2}^{2}}$. Note that $T_{1}$ and $T_{2}$ represent the scattering process at BS1 and BS2, respectively. Also $T_{L}$ corresponds to the optical loss in the system's output field. $D_{1}$ and $D_{2}$ represent the Homodyne measurements with phase $\phi_{1}$ and $\phi_{2}$, respectively.

\subsection{Information gain via entanglement}

This subsection is devoted to show that, in a special setup, an additional information about the system is indeed obtained through the second path in the interferometer, which may improve the control performance. That is, we consider the case where the system's output field completely diminishes, i.e., $\delta=1$. In this case, the $D$ matrix is given by

$$
D=\left[-\beta_{1}\left(\beta_{2} D_{1}+\alpha_{2} D_{2}\right), \alpha_{1}\left(\beta_{2} D_{1}+\alpha_{2} D_{2}\right), \alpha_{2} D_{1}-\beta_{2} D_{2}\right]
$$

and as a result $C=0$ for any choice of $\bar{C}$. Then the system equations (2) and (5) are given by

$$
d \hat{x}_{t}=A \hat{x}_{t} d t+F u_{t} d t+B d \hat{W}_{t}, \quad d y_{t}=D d \hat{W}_{t}
$$

Hence, as expected, the measurement output $y_{t}$ does not explicitly contain any information about the system. However, interestingly, the observer can implicitly gain information; intuitively, this is because the observer knows that the same noise $\hat{W}_{t}$ enters into the system and the detector; in other words, the observer exactly knows 
the noise that drives the system and thus can track the estimate of the system's time evolution. Actually, the quantum Kalman filter equation (7) is now given by

$$
d \pi\left(\hat{x}_{t}\right)=A \pi\left(\hat{x}_{t}\right) d t+F u_{t} d t+B \Re(\Theta) D^{\top}\left(D \Re(\Theta) D^{\top}\right)^{-1} d y_{t},
$$

which means that the observer can update the estimate $\pi\left(\hat{x}_{t}\right)$ using the measurement result $y_{t}$. Also the estimation error covariance matrix follows

$$
\dot{V}_{t}=A V_{t}+V_{t} A^{\top}+B \Re(\Theta) B^{\top}-B \Re(\Theta) D^{\top}\left(D \Re(\Theta) D^{\top}\right)^{-1} D \Re(\Theta) B^{\top}
$$

Now, because $A$ is Hurwitz, $V_{t}$ has a steady solution, meaning that the estimation error is bounded. The above two equations indicate that the important term bringing the information to the filter (19) and (20) is $B \Re(\Theta) D^{\top}$, which is now calculated as

$$
B \Re(\Theta) D^{\top}=\frac{\alpha_{1} \beta_{1}}{2} \Sigma_{n} \bar{C}^{\top} \Sigma_{1}\left[I_{2}-2 \Re\left(\Theta_{1}\right)\right]\left(\beta_{2} D_{1}^{\top}+\alpha_{2} D_{2}^{\top}\right) .
$$

If there is no entanglement (i.e., $r=0$ or $\alpha_{1} \beta_{1}=0$ ), then $B \Re(\Theta) D^{\top}=0$, and the filter equations are reduced to

$$
d \pi\left(\hat{x}_{t}\right)=A \pi\left(\hat{x}_{t}\right) d t+F u_{t} d t, \quad \dot{V}_{t}=A V_{t}+V_{t} A^{\top}+B \Re(\Theta) B^{\top} .
$$

These are simply the dynamics of unconditional expectation $\pi\left(\hat{x}_{t}\right)=\left\langle\hat{x}_{t}\right\rangle$ and the error covariance matrix, which correspond to the master equation describing the statistical time evolution of the system without measurement. Therefore, it is now clear that the entanglement-assisted filter gains additional information about the system through the entangled input field. However, note that an additional information does not always improve the control performance, because, as demonstrated in Sect. 4.5, an entangled probe field is generally fragile and as a result the system's output field becomes more noisy compared to the case of coherent input.

Remark The measurement output $d y_{t}=D d \hat{W}_{t}$ in Eq. (18) has the form of noknowledge measurement [42], which can be used to cancel decoherence. Interestingly, unlike the measurement scheme presented here, the no-knowledge one does not provide any information to the observer; actually for the setup of [42], it can be proven that $A$ is not Hurwitz and the estimation error diverges.

\section{Entanglement-assisted feedback for opto-mechanical oscillator}

In this section, we conduct detailed numerical simulations to evaluate how much the proposed entanglement-assisted feedback control scheme is effective in a practical setup. 


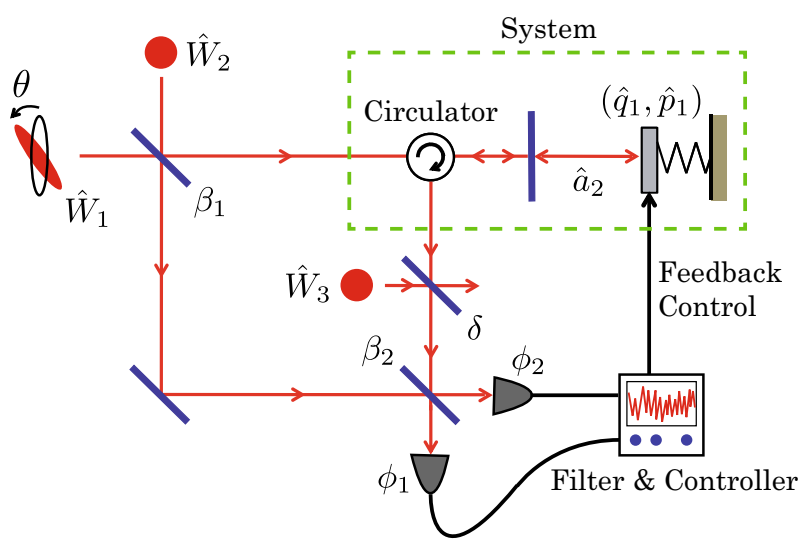

Fig. 3 Opto-mechanical oscillator coupled to the entangled probe field

\subsection{System model}

The system of interest is an opto-mechanical oscillator shown in Fig. 3. Let $\left(\hat{q}_{1}, \hat{p}_{1}\right)$ be the position and momentum operators of the mechanical oscillator, and $\hat{a}_{2}$ be the annihilation operator of the optical cavity. The system Hamiltonian is given by

$$
\hat{H}=\frac{\omega}{2}\left(\hat{q}_{1}^{2}+\hat{p}_{1}^{2}\right)+\frac{\Delta}{2}\left(\hat{q}_{2}^{2}+\hat{p}_{2}^{2}\right)-\lambda \hat{q}_{1} \hat{q}_{2}
$$

where $\omega$ is the resonant frequency of the oscillator and $\Delta$ is the frequency detuning of the cavity mode in the rotating frame of the driving laser frequency; see $[33,36,43]$ for more detailed description. Note $\hat{q}_{2}=\left(\hat{a}_{2}+\hat{a}_{2}^{\dagger}\right) / \sqrt{2}$ and $\hat{p}_{2}=\left(\hat{a}_{2}-\hat{a}_{2}^{\dagger}\right) / \sqrt{2} i$. The third term is the linearized radiation pressure force with strength $|\lambda|$, representing the interaction between the oscillator and the cavity field. From the relation $\hat{H}=\hat{x}^{\top} G \hat{x} / 2$ with $\hat{x}=\left[\hat{q}_{1}, \hat{p}_{1}, \hat{q}_{2}, \hat{p}_{2}\right]^{\top}$, we have

$$
G=\left[\begin{array}{cc|cc}
\omega & 0 & -\lambda & 0 \\
0 & \omega & 0 & 0 \\
\hline-\lambda & 0 & \Delta & 0 \\
0 & 0 & 0 & \Delta
\end{array}\right] .
$$

The system couples to the driving laser field at the partially reflective end-mirror of the cavity, with strength $\kappa$; this coupling is represented by the following operator:

$$
\hat{L}=\sqrt{\kappa} \hat{a}_{2}=\sqrt{\frac{\kappa}{2}}\left(\hat{q}_{2}+i \hat{p}_{2}\right)=\sqrt{\frac{\kappa}{2}}[0,0,1, i] \hat{x} .
$$

Thus, Eq. (15) yields

$$
\bar{C}=\sqrt{\kappa}\left[\begin{array}{ll|ll}
0 & 0 & 1 & 0 \\
0 & 0 & 0 & 1
\end{array}\right] .
$$


This determines the system's $B$ and $C$ matrices from Eqs. (16) and (17), respectively, and the $A$ matrix from Eq. (3). In addition, we assume that the oscillator is subjected to a thermal environment with mean photon number $\bar{n}_{\text {th }}$. Then the system matrices are modified as follows; we need to change the $A$ matrix to $A-\gamma \Gamma / 2$ and the constant term in the Riccati equation (8), $B \Re(\Theta) B^{\top}$, to $B \Re(\Theta) B^{\top}+\gamma\left(\bar{n}_{\text {th }}+1 / 2\right) \Gamma$, where $\gamma$ represents the system-environment coupling strength and $\Gamma=\operatorname{diag}\{1,1,0,0\}$. Note that $A-\gamma \Gamma / 2$ is Hurwitz, meaning that both the Riccati equations (8) and (12) have a unique steady solution. The oscillator can be directly controlled by implementing a piezo-actuator [44] (the case shown in Fig. 3) or indirectly controlled by modulating the input probe field. In both cases, it can be shown that the system satisfies the conditions for the cheap control described in Sect. 2.4; see "Appendix B."

\subsection{Control goal}

The control goal is to cool the oscillator toward its motional ground state; i.e., we want to minimize the stationary mechanical occupation number

$$
\bar{n}=\left\langle\hat{a}_{1, \infty}^{\dagger} \hat{a}_{1, \infty}\right\rangle=\left(\left\langle\hat{q}_{1, \infty}^{2}\right\rangle+\left\langle\hat{p}_{1, \infty}^{2}\right\rangle-1\right) / 2 .
$$

As described in Sect. 2.4, this can be ultimately reduced, by the ideal cheap control, to

$$
\bar{n}^{*}=\left(\operatorname{Tr}\left(\Gamma V_{\infty}\right)-1\right) / 2,
$$

where again $\Gamma=\operatorname{diag}\{1,1,0,0\}$ and $V_{\infty}$ is the steady solution of the Riccati equation (8).

The system parameters are set to the following typical values (in the unit $\omega=1$ ) in the feedback cooling setup (e.g., [36]). First we assume the resonant driving $\Delta=0$, meaning that the oscillator's position and momentum can be best estimated by the filter and accordingly controlled efficiently. Also the cavity line width is set to $\kappa=2$ (bad cavity regime), so that the intra-cavity field immediately leaks to outside and as a consequence the oscillator dynamics can be well observed by the filter. The oscillator is subjected to a thermal noise with mean photon number $\bar{n}_{\text {th }}=1 \times 10^{5}$ with coupling strength $\gamma=1 \times 10^{-7}$. The squeezing level of the probe field is set to $r=2.3$ (10 $\mathrm{dB}$ squeezing), which is accessible with the current technology.

In this setting, the task is to optimize the parameters $\left(\beta_{1}, \beta_{2}, \phi_{1}, \phi_{2}, \theta\right)$ so that Eq. (22) is minimized. To see how to find those optimal parameters, let us assume the lossless setup (i.e., $\delta=0$ ) and focus on only the oscillator mode, where the cavity mode is adiabatically eliminated. This dynamical equation is obtained by setting $d \hat{q}_{2}=0$ and $d \hat{p}_{2}=0$ due to $\kappa \gg \gamma$ and eventually eliminating $\left(\hat{q}_{2}, \hat{p}_{2}\right)$ from the whole dynamical equation;

$$
\begin{aligned}
& d \hat{q}_{1}=-\frac{\gamma}{2} \hat{q}_{1} d t+\omega \hat{p}_{1} d t-\sqrt{\gamma} d \hat{Q}_{\mathrm{th}} \\
& d \hat{p}_{1}=-\omega \hat{q}_{1} d t-\frac{\gamma}{2} \hat{p}_{1} d t+u d t-\frac{2 \lambda}{\sqrt{\kappa}}\left(\alpha_{1} d \hat{Q}_{1}+\beta_{1} d \hat{Q}_{2}\right)-\sqrt{\gamma} d \hat{P}_{\mathrm{th}}
\end{aligned}
$$




$$
\begin{aligned}
d y_{1}= & \frac{2 \alpha_{2} \lambda \sin \phi_{1}}{\sqrt{\kappa}} \hat{q}_{1} d t-\left(\alpha_{1} \alpha_{2}+\beta_{1} \beta_{2}\right)\left(\cos \phi_{1} d \hat{Q}_{1}+\sin \phi_{1} d \hat{P}_{1}\right) \\
& +\left(\alpha_{1} \beta_{2}-\beta_{1} \alpha_{2}\right)\left(\cos \phi_{1} d \hat{Q}_{2}+\sin \phi_{1} d \hat{P}_{2}\right), \\
d y_{2}= & -\frac{2 \beta_{2} \lambda \sin \phi_{2}}{\sqrt{\kappa}} \hat{q}_{1} d t+\left(\alpha_{1} \beta_{2}-\beta_{1} \alpha_{2}\right)\left(\cos \phi_{2} d \hat{Q}_{1}+\sin \phi_{2} d \hat{P}_{1}\right) \\
& +\left(\alpha_{1} \alpha_{2}+\beta_{1} \beta_{2}\right)\left(\cos \phi_{2} d \hat{Q}_{2}+\sin \phi_{2} d \hat{P}_{2}\right)
\end{aligned}
$$

where $u$ represents the magnitude of the force applied to a piezo-actuator mounted on the oscillator and $\left(\hat{Q}_{\mathrm{th}}, \hat{P}_{\mathrm{th}}\right)$ are the quadrature of the thermal field. These equations lead to a rough guide for choosing the parameters, as follows.

1. First, the bigger the first terms in Eqs. (25) and (26) become, the more information the observer gains. Hence, it would be reasonable to make the terms $\sin \phi_{1}$ and $\sin \phi_{2}$ bigger or equivalently take $\phi_{1} \approx \pi / 2$ and $\phi_{2} \approx \pi / 2$.

2. The above choice of $\left(\phi_{1}, \phi_{2}\right)$ implies that the measurement outputs are dominantly affected by the phase quadrature noise $\left(\hat{P}_{1}, \hat{P}_{2}\right)$ rather than the amplitude quadrature noise $\left(\hat{Q}_{1}, \hat{Q}_{2}\right)$. Then by squeezing $\hat{P}_{1}$, we can improve the signal-to-noise ratio in both $y_{1}$ and $y_{2}$. This means that $\theta \approx \pi / 2$ would be a proper choice.

3. The coefficients of the noise terms related to $\hat{W}_{1}=\left[\hat{Q}_{1}, \hat{P}_{1}\right]^{\top}$ and $\hat{W}_{2}=$ $\left[\hat{Q}_{2}, \hat{P}_{2}\right]^{\top}$ in $y_{1}$ and $y_{2}$ cannot be simultaneously reduced, because they satisfy $\left(\alpha_{1} \alpha_{2}+\beta_{1} \beta_{2}\right)^{2}+\left(\alpha_{1} \beta_{2}-\beta_{1} \alpha_{2}\right)^{2}=1$. Then, because $\hat{W}_{2}$ is not a tunable noise field, meaning that $\left\langle\left(\cos \phi_{1} d \hat{Q}_{2}+\sin \phi_{1} d \hat{P}_{2}\right)^{2}\right\rangle=d t$ and $\left\langle\left(\cos \phi_{2} d \hat{Q}_{2}+\sin \phi_{2} d \hat{P}_{2}\right)^{2}\right\rangle=d t$, it would be reasonable to choose the BS parameters so that the coefficient of $\hat{W}_{2}$ is reduced; more precisely, $\alpha_{1} \beta_{2}-\beta_{1} \alpha_{2} \approx$ 0 if $y_{1}$ is mainly used (i.e., $\alpha_{2} \approx 1$ ), or $\alpha_{1} \alpha_{2}+\beta_{1} \beta_{2} \approx 0$ if $y_{2}$ is mainly used (i.e., $\left.\beta_{2} \approx 1\right)$. This leads to $\left(\alpha_{1}, \beta_{1}, \alpha_{2}, \beta_{2}\right) \approx(1,0,1,0)$ or $\left(\alpha_{1}, \beta_{1}, \alpha_{2}, \beta_{2}\right) \approx$ $(1,0,0,1)$.

Of course, the above intuitive observation, particularly the last one, would not be necessarily true. In fact, we have now arrived at $\left(\alpha_{1}, \beta_{1}\right) \approx(1,0)$, but this means that the input probe field is nearly a separable state where the squeezed component is injected to the system, and the entanglement property is not effectively used. Moreover, when $\left(\alpha_{1}, \beta_{1}\right) \approx(1,0)$, the back-action noise on $\hat{p}_{1}$ (i.e., the fourth term in the righthand side of Eq. (24)) is dominated by $d \hat{Q}_{1}$; however, because now $\hat{Q}_{1}$ is nearly anti-squeezed (due to $\theta \approx \pi / 2$ ), this parameter choice induces a bigger back-action noise. Therefore, the parameters have to be carefully chosen, via detailed numerical simulations taking into account the trade-off between the back-action noise and the signal-to-noise ratio for the measurement outputs $y_{1}$ and $y_{2}$.

\subsection{Effectiveness of the entanglement-assisted feedback control}

First, let us see if the entanglement would actually bring any advantage to the feedback control. Figure 4a shows $\bar{n}^{*}$ as a function of the reflectivity of BS1, $\beta_{1}^{2}$, in the case $\lambda=0.3$ (weak coupling regime) and $\delta=0$ (the system's output field has no loss). $\bar{n}^{*}$ is calculated from Eq. (22) together with the steady solution $V_{\infty}$ of the Riccati equation (8). Furthermore, it is minimized with respect to the phase of the probe 
(a)

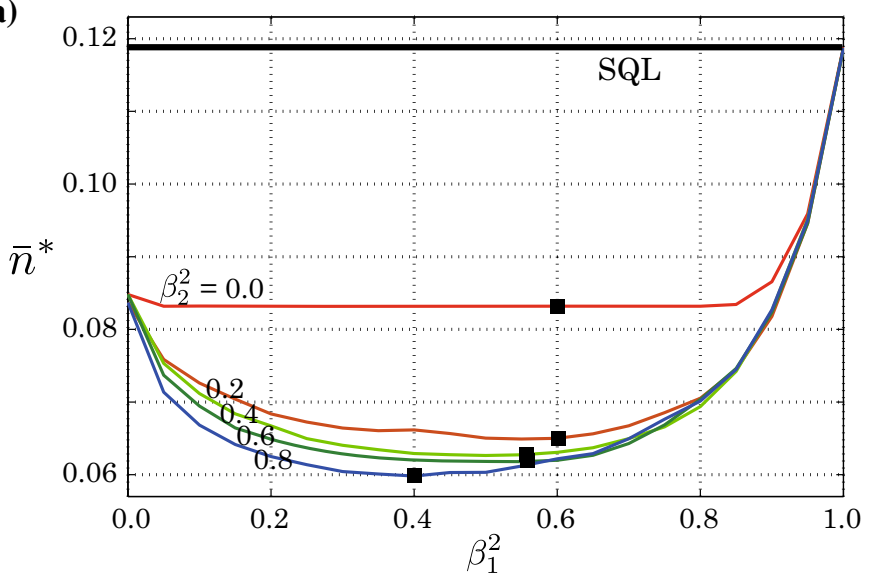

(b)

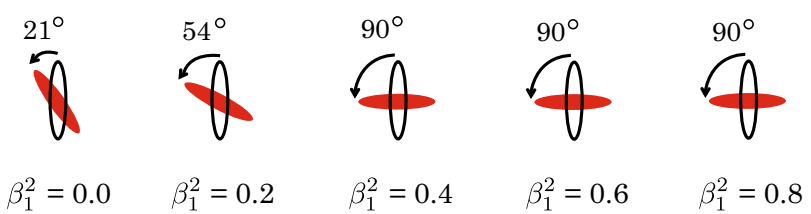

Fig. 4 a Achievable lowest mechanical occupation number, $\bar{n}^{*}$, versus $\beta_{1}^{2}$ (the reflectivity of BS1) for several values of $\beta_{2}^{2}$, for the case $\lambda=0.3$ and $\delta=0$. At each point of $\beta_{1}$, the phase of the squeezed field and the Homodyne detection, $\left(\theta, \phi_{1}, \phi_{2}\right)$, are optimized. The black box indicates the minimum of $\bar{n}^{*}$. b The optimal phase $\theta$ of the squeezed field at each $\beta_{1}$ for the case $\beta_{2}^{2}=0.8$

squeezed field, $\theta$, and the phases of the two Homodyne detectors, $\left(\phi_{1}, \phi_{2}\right)$, at each $\beta_{1}$; Fig. $4 \mathrm{~b}$ illustrates the optimal $\theta$ at each $\beta_{1}$ for the case $\beta_{2}^{2}=0.8$. The reflectivity $\beta_{1}^{2}$ represents how much the squeezed field $\hat{W}_{1}$ is split into two arms, which determines the amount of entanglement. Note that when $\beta_{1}=0$ or $\beta_{1}=1$, the input fields are not entangled. In particular, $\beta_{1}=1$ corresponds to the standard case where only the coherent field is injected to the system; hence, the value of $\bar{n}^{*}$ in this case has the meaning of SQL, which is now $\bar{n}_{\mathrm{SQL}}^{*} \approx 0.119$ as indicated in Fig. 4a. The five solid curves in Fig. 4a show $\bar{n}^{*}$ for several values of $\beta_{2}^{2}$, the reflectivity of BS2; recall that $\beta_{2}=0$ means the case of local measurement, while the cases $\beta_{2} \neq 0$ correspond to the global measurement (see (iv) in Sect. 3.1). Importantly, in all cases the minimum of $\bar{n}^{*}$, which is indicated by the black box, is smaller than the SQL and is attained at a certain point of $\beta_{1} \in(0,1)$, where the input field is entangled. In particular, the most effective feedback cooling is carried out when we use the highly entangled probe field with $\beta_{1}^{2}=0.4$ and perform the global measurement with $\beta_{2}^{2}=0.8$, in which case the minimum of $\bar{n}^{*}$ is about 0.06 . As a conclusion, the entanglement-assisted feedback control is in fact effective and realizes further cooling of the oscillator below the SQL. The followings are the list of other notable features of this system.

- For the cases $\beta_{1}^{2}=0.4,0.6,0.8$, the optimal phase of the squeezed field is $\theta=\pi / 2$. The optimality of $\theta=\pi / 2$ was indeed expected in the second observation in 
Sect. 4.2 (page 13), but $\beta_{1}$ is not nearly zero, which is not consistent with the third observation in Sect. 4.2. Therefore, the numerical solver has actually chosen a non-trivial set of parameters that balances the back-action noise on $\hat{p}_{1}$ and the measurement noise on $\left(y_{1}, y_{2}\right)$.

- The minimum of $\bar{n}^{*}$ is reached at $\beta_{1}^{2} \neq 0.5$ and $\theta=\pi / 2$. This means that the maximal entangled field is not the best probe for the estimation and feedback control; see "Appendix C."

- The entanglement-assisted method outperforms the control with the optimized squeezed probe field [13], which corresponds to the case $\beta_{1}=0$.

\subsection{Coupling strength and optimal probe}

Here we study how much the minimum occupation number $\bar{n}_{\min }^{*}$ changes with respect to the coupling strength $\lambda$. Figure 5 shows $\bar{n}^{*}$ as a function of $\theta$ and $\beta_{2}$, for $\delta=0$ and several values of $\lambda$ : (a) $\lambda=0.3$, (b) $\lambda=0.5$, (c) $\lambda=0.7$, and (d) $\lambda=0.9$. In each Fig. $5 \mathrm{a}-\mathrm{d}, \bar{n}^{*}$ is already minimized with respect to $\left(\beta_{1}, \phi_{1}, \phi_{2}\right)$. In particular, in each figure, the optimal value of $\beta_{1}$ has been chosen as: (a) $\beta_{1}^{2}=0.40$, (b) $\beta_{1}^{2}=0.65$, (c) $\beta_{1}^{2}=0.55$, and (d) $\beta_{1}^{2}=0.50$, implying that the input probe field is highly entangled in all cases. Hence, we end up with the same conclusion that the entanglement-assisted

(a)

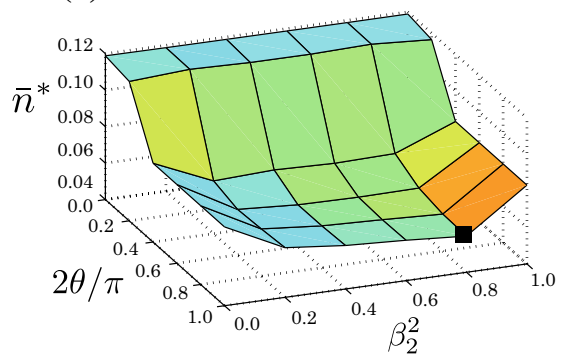

(c)

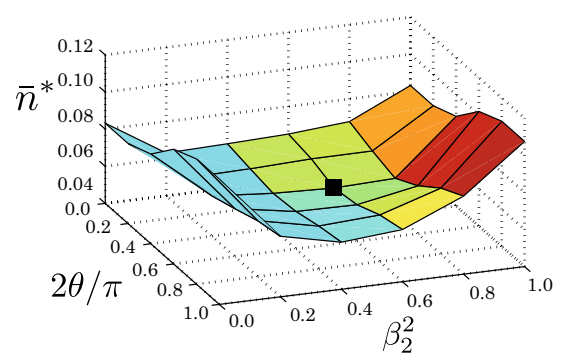

(b)

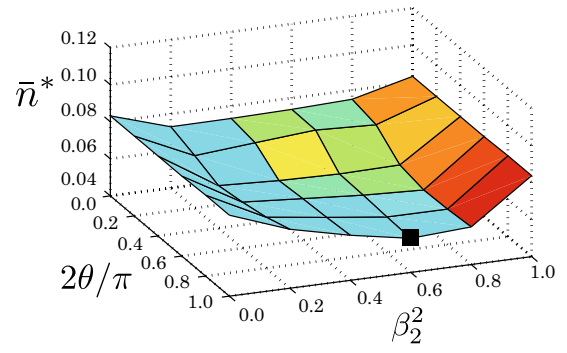

(d)

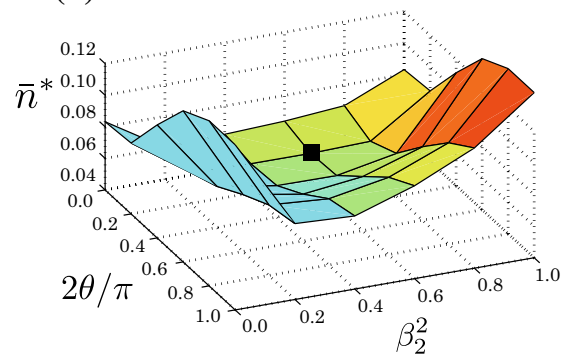

Fig. 5 Achievable lowest mechanical occupation number, $\bar{n}^{*}$, as a function of $\theta$ (phase of the squeezed field) and $\beta_{2}^{2}$ (reflectivity of BS2), for $\delta=0$ and several values of $\lambda$ (strength of the radiation pressure): a $\lambda=0.3, \mathbf{b} \lambda=0.5$, $\mathbf{c} \lambda=0.7$, and $\mathbf{d} \lambda=0.9$. The black box indicates the minimum of $\bar{n}^{*}$. The achieved $\bar{n}_{\min }^{*}$ and the SQL $\bar{n}_{\mathrm{SQL}}^{*}$ are respectively a $(0.060,0.119), \mathbf{b}(0.058,0.084), \mathbf{c}(0.058,0.018), \mathbf{d}(0.060,0.145)$ 
feedback control cools the oscillator below the SQL and even performs better than the case with optimized squeezed probe field.

Note here that, as implied by Eqs. (24), (25), and (26), making $\lambda$ bigger improves the signal-to-noise ratio in the measurement output, but at the same time this induces a bigger back-action noise on $\hat{p}_{1}$. Hence, $\bar{n}_{\mathrm{SOL}}^{*}$ does not monotonically change with respect to $\lambda$; interestingly, $\bar{n}_{\text {min }}^{*}$ takes almost the same value for all $\lambda$, which suggests that there would exist a fundamental lower bound of $\bar{n}_{\min }^{*}$ that is independent to $\lambda$. Another remarkable fact is that, for small values of $\lambda$, the optimal phase of the squeezed field is $\theta=\pi / 2$, as seen in the previous subsection; however, this does not hold when $\lambda$ becomes large. This is because, for a large $\lambda$, it is more important to reduce the back-action noise $(2 \lambda / \sqrt{\kappa}) \alpha_{1} d \hat{Q}_{1}$ than to improve the signal-to-noise ratio in the measurement process, and thus, the squeezed field with $\left\langle d \hat{Q}_{1}^{2}\right\rangle\left\langle\left\langle d \hat{P}_{1}^{2}\right\rangle\right.$ is chosen.

\subsection{The case of lossy output field}

Next let us consider the case where the system's output field is subjected to the optical loss. Figure 6 shows the plot of $\bar{n}^{*}$ with the same setting as in Fig. 4 (i.e., $\lambda=0.3$ and $\left(\theta, \phi_{1}, \phi_{2}\right)$ are optimized), except that the loss parameter is now set to $\delta^{2}=0.1$. As in the lossless case $\delta=0$, we find that the minimum of $\bar{n}^{*}$ is reached when the input probe field is entangled $\left(\beta_{1}^{2} \approx 0.8\right)$ and the global measurement $\left(\beta_{2}^{2}=0.8\right)$ is performed. However, notably, the difference between the minimum value of $\bar{n}^{*}$ and the SQL given at $\beta_{1}=1$ (i.e., how much the control performance is improved by entanglement) is smaller than the case when $\delta=0$. That is, the entanglement-assisted feedback is less effective if the system's output field is lossy. Another notable feature is that there is a case where the control performance becomes worse than the SQL via the entanglement-assisted feedback control. This happens when $\beta_{1}$ takes a small value, in which case the portion injected into the system is nearly a pure squeezed

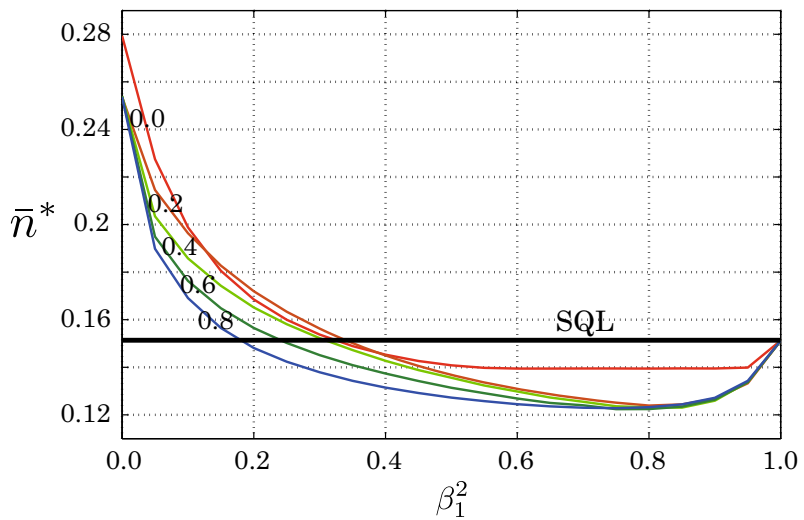

Fig. 6 Achievable lowest mechanical occupation number, $\bar{n}^{*}$, versus $\beta_{1}^{2}$ (the reflectivity of BS1) for several values of $\beta_{2}^{2}$, for the case $\lambda=0.3$ and $\delta^{2}=0.1$. At each point of $\beta_{1}$, the phase of the squeezed light field and the Homodyne detection, $\left(\theta, \phi_{1}, \phi_{2}\right)$, are optimized. The numbers indicated along the curves are the values of $\beta_{2}^{2}$, as in the case of Fig. 4 
(a)

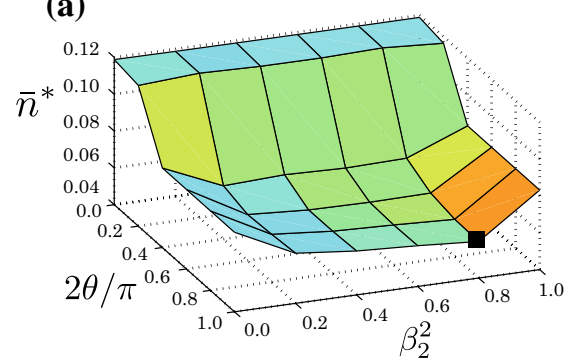

(c)

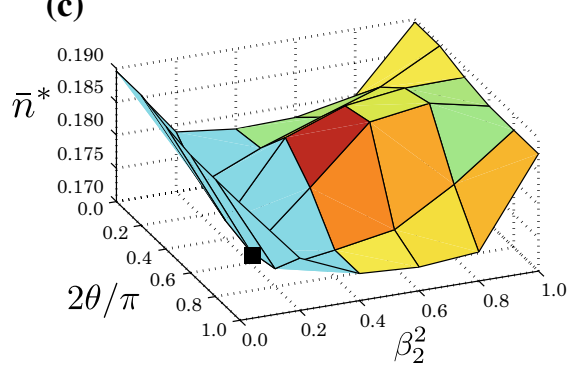

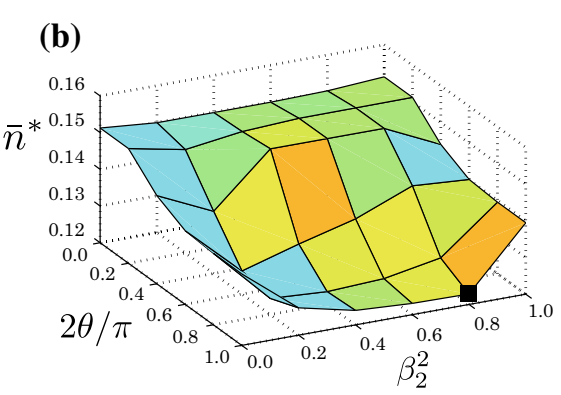

(d)

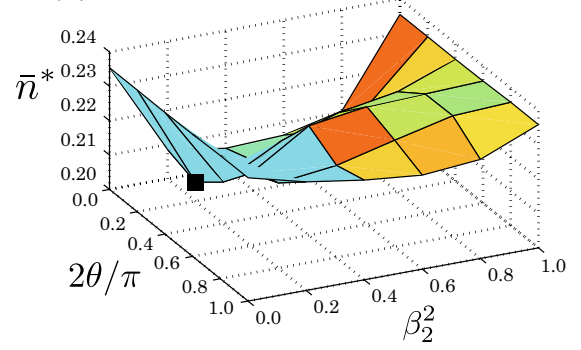

Fig. 7 Achievable lowest mechanical occupation number, $\bar{n}^{*}$, as a function of $\theta$ (phase of the squeezed light field) and $\beta_{2}^{2}$ (reflectivity of BS2), for $\lambda=0.3$ and several values of $\delta$ (loss in the system's output field): $\mathbf{a} \delta^{2}=0.0, \mathbf{b} \delta^{2}=0.1, \mathbf{c} \delta^{2}=0.2$, and $\mathbf{d} \delta^{2}=0.3$. The black box indicates the minimum of $\bar{n}^{*}$. The achieved $\bar{n}_{\min }^{*}$ and the SQL $\bar{n}_{\mathrm{SQL}}^{*}$ are respectively a $(0.060,0.119), \mathbf{b}(0.123,0.151), \mathbf{c}(0.172,0.190), \mathbf{d}$ $(0.201,0.236)$

field. This result makes sense, because, as is well known, a squeezed field is fragile to noise and the system's output field loses more information than the standard case, which cannot be compensated by the additional information gained from the second path of the interferometer.

Finally, Fig. 7 shows the plot of $\bar{n}^{*}$ as a function of $\theta$ and $\beta_{2}$, for $\lambda=0.3$ and several values of $\delta$ : (a) $\delta^{2}=0.0$, (b) $\delta^{2}=0.1$, (c) $\delta^{2}=0.2$, and (d) $\delta^{2}=0.3$. As in the case of Fig. $5, \bar{n}^{*}$ is already minimized with respect to $\left(\beta_{1}, \phi_{1}, \phi_{2}\right)$. Note that Fig. 7 a is the same as Fig. 5a. A notable point is that the optimal values of $\theta$ and $\beta_{2}$ in the case $\delta^{2}=0.1$ are the same as those for $\delta=0$. This means that the optimal input probe field and measurement are independent to the system's output loss $\delta$. This is a desirable fact because an exact value of $\delta$ is hard to estimate in practice, but the same input probe field and measurement can be used without respect to $\delta$ as long as the system's output loss is enough suppressed. However, Fig. 7c, d shows that the probe and measurement have to be changed when $\delta$ becomes bigger. In this sense, the optimal probe field is not robust for a system with lossy output.

\section{Conclusion}

In this paper, we have formulated the entanglement-assisted feedback control method for general linear quantum systems, which involves optimization of the amount of 
entanglement, the phase of the probe squeezed field, and the Homodyne measurement. Thanks to the linear setting, the strict lower bound of LQG cost function, which is achievable by the ideal cheap control, can be explicitly obtained, and it is used to evaluate the control performance. In the detailed numerical simulation studying the cooling problem of an opto-mechanical oscillator, it was shown that the entanglementassisted controller works better than the standard method without entanglement, i.e., the control with a coherent probe field and even that with an optimized squeezed probe field. Although the improvement is not so drastic especially when the system's output field is lossy, we expect that a significant advantage of the entanglement-assisted method would appear for some nonlinear systems. In fact, it was shown in [45] that, in a different measurement configuration, an entangled probe field can be used to significantly improve the detection efficiency for a qubit system; an extension of this study to the feedback control problem is an interesting future work.

Open Access This article is distributed under the terms of the Creative Commons Attribution 4.0 International License (http://creativecommons.org/licenses/by/4.0/), which permits unrestricted use, distribution, and reproduction in any medium, provided you give appropriate credit to the original author(s) and the source, provide a link to the Creative Commons license, and indicate if changes were made.

\section{Appendix A: Solution of LQG control problem}

Here we briefly explain how to derive the solution of the LQG control problem; see [40] for a more detailed derivation. The essential idea is to use the dynamic programming method based on the following expected cost-to-go:

$$
J_{t}[u, z]=\mathbb{E}\left[\int_{t}^{T}\left(\pi\left(\hat{x}_{s}\right)^{\top} Q \pi\left(\hat{x}_{s}\right)+u_{s}^{\top} R u_{s}\right) \mathrm{d} s \mid \pi\left(\hat{x}_{t}\right)=z\right] .
$$

The goal is to obtain the minimum of this function, i.e., $J_{t}^{*}(z)=\min _{u[t, T]} J_{t}[u, z]$, with respect to the input in the time interval $[t, T]$, denoted by $u[t, T]$. Now we rewrite $J_{t}^{*}(z)$ in the following form:

$$
\begin{aligned}
J_{t}^{*}(z)= & \min _{u[t, T]} \mathbb{E}\left[\int_{t}^{t+d t}\left(\pi\left(\hat{x}_{s}\right)^{\top} Q \pi\left(\hat{x}_{s}\right)+u_{s}^{\top} R u_{s}\right) \mathrm{d} s\right. \\
& \left.\quad+\int_{t+d t}^{T}\left(\pi\left(\hat{x}_{s}\right)^{\top} Q \pi\left(\hat{x}_{s}\right)+u_{s}^{\top} R u_{s}\right) \mathrm{d} s \mid \pi\left(\hat{x}_{t}\right)=z\right] \\
= & \min _{u_{t}}\left\{\left(z^{\top} Q z+u_{t}^{\top} R u_{t}\right) d t+J_{t+d t}^{*}(z+d z)\right\} .
\end{aligned}
$$

Then, noting that $\pi\left(\hat{x}_{t}\right)$ obeys Eq. (7) and $d \bar{w}_{t}=d y_{t}-C \pi\left(\hat{x}_{t}\right) d t$ is the standard classical Wiener process satisfying $d \bar{w}_{t} d \bar{w}_{t}^{\top}=D \Theta D^{\top} d t$, we see that the optimal value function $J_{t}^{*}(z)$ satisfies the Bellman equation

$$
\min _{u_{t}}\left\{\left\|u_{t}+\frac{1}{2} R^{-1} F^{\top} \frac{\partial J_{t}^{*}(z)}{\partial z}\right\|_{R}^{2}-\frac{1}{4}\left(\frac{\partial J_{t}^{*}(z)}{\partial z}\right)^{\top} F R^{-1} F^{\top} \frac{\partial J_{t}^{*}(z)}{\partial z}+\frac{\partial J_{t}^{*}(z)}{\partial t}\right.
$$




$$
\left.+z^{\top} Q z+\left(\frac{\partial J_{t}^{*}(z)}{\partial z}\right)^{\top} A z+\frac{1}{2} \operatorname{Tr}\left[\frac{\partial^{2} J_{t}^{*}(z)}{\partial z^{2}} K_{t} D \Re(\Theta) D^{\top} K_{t}^{\top}\right]\right\}=0,
$$

with the terminal condition $J_{T}^{*}(z)=0$. Here we have defined $\|x\|_{R}^{2}=x^{\top} R x$. The optimal control input is thus given by

$$
u_{t}^{*}=-\frac{1}{2} R^{-1} F^{\top} \frac{\partial J_{t}^{*}(z)}{\partial z}
$$

The optimal value function $J_{t}^{*}(z)$ is then determined from the following partial differential equation:

$$
\begin{aligned}
& \frac{\partial J_{t}^{*}(z)}{\partial t}+z^{\top} Q z-\frac{1}{4}\left(\frac{\partial J_{t}^{*}(z)}{\partial z}\right)^{\top} F R^{-1} F^{\top} \frac{\partial J_{t}^{*}(z)}{\partial z}+\left(\frac{\partial J_{t}^{*}(z)}{\partial z}\right)^{\top} A z \\
& +\frac{1}{2} \operatorname{Tr}\left[\frac{\partial^{2} J_{t}^{*}(z)}{\partial z^{2}} K_{t} D \Re(\Theta) D^{\top} K_{t}^{\top}\right]=0 .
\end{aligned}
$$

Now we assume that the solution is of the quadratic form $J_{t}^{*}(z)=z^{\top} P_{t} z+v_{t}$ with $P_{t} \in \mathbb{R}^{2 n \times 2 n}$ and $\nu_{t} \in \mathbb{R}$; then the above partial differential equation is reduced to

$$
\begin{aligned}
& z^{\top}\left(\dot{P}_{t}+P_{t} A+A^{\top} P_{t}-P_{t} F R^{-1} F^{\top} P_{t}+Q\right) z \\
& \quad+\dot{v}_{t}+\operatorname{Tr}\left[P_{t} K_{t} D \Re(\Theta) D^{\top} K_{t}^{\top}\right]=0 .
\end{aligned}
$$

This equality must hold for any $z \in \mathbb{R}^{2 n}$, and we thus obtain the following set of ordinary differential equations:

$\dot{P}_{t}+P_{t} A+A^{\top} P_{t}-P_{t} F R^{-1} F^{\top} P_{t}+Q=0, \quad \dot{v}_{t}+\operatorname{Tr}\left[P_{t} K_{t} D \Re(\Theta) D^{\top} K_{t}^{\top}\right]=0$.

It follows from $J_{T}^{*}(z)=0$ that the terminal conditions are $P_{T}=0$ and $v_{T}=0$. Under the assumption that the above set of equations have solutions, the optimal controller (27) is given by

$$
u_{t}^{*}=-R^{-1} F^{\top} P_{t} \pi\left(\hat{x}_{t}\right)
$$

Moreover, we now have

$$
\left\langle J_{0}^{*}\left(\hat{x}_{0}\right)\right\rangle=\left\langle\hat{x}_{0}^{\top} P_{0} \hat{x}_{0}+v_{0}\right\rangle=\left\langle\hat{x}_{0}^{\top} P_{0} \hat{x}_{0}\right\rangle+\int_{0}^{T} \operatorname{Tr}\left[P_{t} K_{t} D \Re(\Theta) D^{\top} K_{t}^{\top}\right] \mathrm{d} t
$$

hence, the minimum of the original cost function (13) is given by

$$
J\left[u^{*}\right]=\lim _{T \rightarrow \infty} \frac{1}{T}\left\langle J_{0}^{*}\left(\hat{x}_{0}\right)\right\rangle+\operatorname{Tr}\left(Q V_{\infty}\right)=\operatorname{Tr}\left[P_{\infty} K_{\infty} D \Re(\Theta) D^{\top} K_{\infty}^{\top}\right]+\operatorname{Tr}\left(Q V_{\infty}\right)
$$




\section{Appendix B: Condition for the cheap control [30-32]}

Let us consider the system whose transfer function matrix is given by $\Xi(s)=\bar{Q}(s I-$ $A)^{-1} F$, which we simply call the system $(A, F, \bar{Q})$. First, if there exist a complex number $z \in \mathbb{C}$ and a vector $u$ such that $u^{\top} G(z)=0$ or $G(z) u=0$, then $z$ is called a zero (more precisely, it is called a transmission zero). Then the system $(A, F, \bar{Q})$ is called minimum phase, if all the zeros of $\Xi(s)$ have negative real part. Next, the system $(A, F, \bar{Q})$ is called right invertible, if $\Xi(s)$ has full row rank for at least one $s \in \mathbb{C}$. In general, such a minimum phase and right invertible system are regarded as a system easy to control; an intuitive understanding of this fact is that there exists an "inverse" and "stable" system (i.e., there exists $\Xi(s)^{-1}$, and all its poles have negative real part), and this system completely compensates $\Xi(s)$. In fact, as mentioned in Sect. 2.4, for such a system there exists a stabilizing controller that well suppresses the dynamical fluctuation of the (estimated) system variables.

Here we prove that the opto-mechanical system examined in Sect. 4 actually satisfies the above condition for cheap control. Note that the LQG problem is now formulated with the choice

$$
\bar{Q}=\left[\begin{array}{llll}
1 & 0 & 0 & 0 \\
0 & 1 & 0 & 0
\end{array}\right],
$$

which actually yields $Q=\bar{Q}^{\top} \bar{Q}=\operatorname{diag}\{1,1,0,0\}$; see Eq. (22). The $F$ matrix representing the actuator mechanism of the controller can be typically chosen as follows. First, if the oscillator can be directly manipulated via a piezoelectrical device, then $F_{1}=[0,1,0,0]^{\top}$, meaning that the momentum of the oscillator can be driven by an external force. Another typical setup for actuation is that the control is carried out by modulating the input probe field, in which case $F_{2}=[0,0,1,0]^{\top}$, where especially only the $\hat{q}_{2}$ quadrature is assumed to be modulated (it can be proven that modulating $\hat{p}_{2}$ does not affect on the condition to be fulfilled). Then we have

$$
\begin{aligned}
& \Xi_{1}(s)=\bar{Q}(s I-A)^{-1} F_{1}=\frac{1}{(s+\kappa / 2)^{2}+\omega^{2}}\left[\begin{array}{c}
\omega \\
s+\gamma / 2
\end{array}\right] \\
& \Xi_{2}(s)=\bar{Q}(s I-A)^{-1} F_{2}=\frac{\lambda}{\left[(s+\kappa / 2)^{2}+\omega^{2}\right](s+\kappa / 2)}\left[\begin{array}{c}
\omega \\
s+\gamma / 2
\end{array}\right] .
\end{aligned}
$$

Therefore, in both cases, the system $(A, F, \bar{Q})$ is minimum phase and right invertible.

\section{Appendix C: Logarithmic negativity}

For a two-mode Gaussian state with mean zero, its correlation property can be completely characterized by the covariance matrix

$$
V=\left[\begin{array}{cc}
V_{1} & V_{2} \\
V_{2}^{\top} & V_{3}
\end{array}\right],
$$


where $V_{i}$ are $2 \times 2$ matrices. In particular, the following logarithmic negativity $[46,47]$ can be used as a reasonable measure of entanglement of this Gaussian state:

$$
E_{\mathcal{N}}=\max \{0,-\log (2 v)\}
$$

where $\log x$ denotes the natural logarithm of $x$, and

$$
v=\frac{1}{\sqrt{2}} \sqrt{\tilde{\Delta}-\sqrt{\tilde{\Delta}^{2}-4 \operatorname{det}(V)}}, \quad \tilde{\Delta}=\operatorname{det}\left(V_{1}\right)+\operatorname{det}\left(V_{3}\right)-2 \operatorname{det}\left(V_{2}\right)
$$

Actually the state is entangled if and only if $E_{\mathcal{N}}>0$.

In our case, the output of BS1 is an entangled Gaussian field; particularly when $\theta=\pi / 2$, the covariance (more precisely the spectral density) matrix is given by Eq. (29) with

$$
\begin{aligned}
& V_{1}=\operatorname{diag}\left\{\alpha_{1}^{2} e^{r}+\beta_{1}^{2}, \alpha_{1}^{2} e^{-r}+\beta_{1}^{2}\right\} / 2, \\
& V_{2}=\operatorname{diag}\left\{\alpha_{1} \beta_{1}\left(1-e^{r}\right), \alpha_{1} \beta_{1}\left(1-e^{-r}\right)\right\} / 2, \\
& V_{3}=\operatorname{diag}\left\{\beta_{1}^{2} e^{r}+\alpha_{1}^{2}, \beta_{1}^{2} e^{-r}+\alpha_{1}^{2}\right\} / 2 .
\end{aligned}
$$

This yields $v=\sqrt{d-\sqrt{d^{2}-1}} / 2$ with $d=2 \alpha_{1}^{2} \beta_{1}^{2}\left(e^{r}+e^{-r}-2\right)+1$, and thus, $E_{\mathcal{N}}>0$ for all $\beta_{1} \in(0,1)$ and $r \neq 0$. Note that, hence, the maximal entangled field for $\theta=\pi / 2$ is produced when $\alpha_{1}^{2}=\beta_{1}^{2}=1 / 2$.

\section{References}

1. Einstein, A., Podolsky, B., Rosen, N.: Can quantum-mechanical description of physical reality be considered complete? Phys. Rev. 47, 777 (1935)

2. Nielsen, M.A., Chuang, I.L.: Quantum Computation and Quantum Information. Cambridge University Press, Cambridge (2000)

3. Dowling, J.P., Milburn, G.J.: Quantum technology: the second quantum revolution. Philos. Trans. R. Soc. Lond. A 361, 1655-1674 (2003)

4. Braunstein, S.L., Caves, C.M.: Statistical distance and the geometry of quantum states. Phys. Rev. Lett. 72, 3439 (1994)

5. Giovannetti, V., Lloyd, S., Maccone, L.: Quantum-enhanced measurements: beating the standard quantum limit. Science 306, 1330 (2004)

6. Mitchell, M.W., Lundeen, J.S., Steinberg, A.M.: Super-resolving phase measurements with a multiphoton entangled state. Nature 429, 161-164 (2004)

7. Nagata, T., Okamoto, R., O’Brien, J.L., Sasaki, K., Takeuchi, S.: Beating the standard quantum limit with four-entangled photons. Science 316, 726-729 (2007)

8. Appel, L., Windpassinger, P.J., Oblak, D., Busk, H.U., Kjargaard, N., Polzik, E.S.: Mesoscopic atomic entanglement for precision measurements beyond the standard quantum limit. Proc. Natl. Acad. Sci. 106, 10960-10965 (2009)

9. Chauvet, A.L., Appel, J., Renema, J.J., Oblak, D., Kjargaard, N., Polzik, E.S.: Entanglement-assisted atomic clock beyond the projection noise limit. New J. Phys. 12(6), 065032 (2010)

10. Kimble, H.J., Levin, Y., Matsko, A.B., Thorne, K.S., Vyatchanin, S.P.: Conversion of conventional gravitational-wave interferometers into quantum nondemolition interferometers by modifying their input and/or output optics. Phys. Rev. D 65, 022002 (2001)

11. Aasi, J., et al.: Enhanced sensitivity of the LIGO gravitational wave detector by using squeezed states of light. Nat. Photon. 7, 613 (2013) 
12. Iwasawa, K., Makino, K., Yonezawa, H., Tsang, M., Davidovic, A., Huntington, E., Furusawa, A.: Quantum-limited mirror-motion estimation. Phys. Rev. Lett. 111, 163602 (2013)

13. Schafermeier, C., et al.: Quantum enhanced feedback cooling of a mechanical oscillator using nonclassical light. Nat. Commun. 7, 13628 (2016)

14. Vyatchanin, S.P., Zubova, E.A.: Quantum variation measurement of a force. Phys. Lett. A 201, 269-274 (1995)

15. Wiseman, H.M., Milburn, G.J.: Quantum Measurement and Control. Cambridge University Press, Cambridge (2009)

16. Jacobs, K.: Quantum Measurement Theory and Its Applications. Cambridge University Press, Cambridge (2014)

17. Belavkin, V.P.: Measurement, filtering and control in quantum open dynamical systems. Rep. Math. Phys. 43, 405-425 (1999)

18. Bouten, L., van Handel, R., James, M.: An introduction to quantum filtering. SIAM J. Control Optim. 46, 2199-2241 (2007)

19. Genoni, M.G., Mancini, S., Wiseman, H.M., Serafini, A.: Quantum filtering of a thermal master equation with purified reservoir. Phys. Rev. A 90, 063826 (2014)

20. Bachor, H.A., Ralph, T.C.: A Guide to Experiments in Quantum Optics. Wiley-VCH, Weinheim (2004)

21. James, M.R., Nurdin, H.I., Petersen, I.R.: $H^{\infty}$ control of linear quantum stochastic systems. IEEE Trans. Autom. Control 53(8), 1787-1803 (2008)

22. Nurdin, H.I., James, M.R., Petersen, I.R.: Coherent quantum LQG control. Automatica 45, 1837 (2009)

23. Hamerly, R., Mabuchi, H.: Advantages of coherent feedback for cooling quantum oscillators. Phys. Rev. Lett. 109, 173602 (2012)

24. Yamamoto, N.: Coherent versus measurement feedback: linear systems theory for quantum information. Phys. Rev. X 4, 041029 (2014)

25. Yamamoto, N.: Quantum feedback amplification. Phys. Rev. Appl. 5, 044012 (2016)

26. Furusawa, A., van Loock, P.: Quantum Teleportation and Entanglement: A Hybrid Approach to Optical Quantum Information Processing. Wiley-VCH, Berlin (2011)

27. Doherty, A.C., Tan, S.M., Parkins, A.S., Walls, D.F.: State determination in continuous measurement. Phys. Rev. A 60, 2380 (1999)

28. Doherty, A.C., Jacobs, K.: Feedback control of quantum systems using continuous state estimation. Phys. Rev. A 60, 2700 (1999)

29. Belavkin, V.P., Edwards, S.C.: Quantum filtering and optimal control. In: Belavkin, V.P., Guta, M. (eds.) Quantum Stochastics and Information: Statistics, Filtering and Control (University of Nottingham, UK, 15-22 July 2006), pp. 143-205. World Scientific, Singapore (2008)

30. Kwakernaak, H., Sivan, R.: The maximal achievable accuracy of linear optimal regulators and linear optimal filters. IEEE Trans. Autom. Control 17, 79-86 (1972)

31. Seron, M.M., Braslavsky, J., Goodwin, G.: Fundamental Limitations in Filtering and Control. Springer, New York (1997)

32. Seron, M.M., Braslavsky, J.H., Kokotovic, P.V., Mayne, D.Q.: Feedback limitations in nonlinear systems: from Bode integrals to cheap control. IEEE Trans. Autom. Control 44(4), 829-833 (1999)

33. Mancini, S., Vitali, D., Tombesi, P.: Optomechanical cooling of a macroscopic oscillator by homodyne feedback. Phys. Rev. Lett. 80, 688 (1998)

34. Hopkins, A., Jacobs, K., Habib, S., Schwab, K.: Feedback cooling of a nanomechanical resonator. Phys. Rev. B 68, 235328 (2003)

35. Hamerly, R., Mabuchi, H.: Coherent controllers for optical-feedback cooling of quantum oscillators. Phys. Rev. A 87, 013815 (2013)

36. Hofer, S.G., Hammerer, K.: Entanglement-enhanced time-continuous quantum control in optomechanics. Phys. Rev. A 91, 033822 (2015)

37. Wilson, D.J., Sudhir, V., Piro, N., Schilling, R., Ghadimi, A., Kippenberg, T.J.: Measurement-based control of a mechanical oscillator at its thermal decoherence rate. Nature 524, 325 (2015)

38. Wieczorek, W., Hofer, S.G., Hoelscher-Obermaier, J., Riedinger, R., Hammerer, K., Aspelmeyer, M.: Optimal state estimation for cavity optomechanical systems. Phys. Rev. Lett. 114, 223601 (2015)

39. Kucera, V.: A contribution to matrix quadratic equation. IEEE Trans. Autom. Control 17(3), 344-347 (1972)

40. Bensoussan, A.: Stochastic Control of Partially Observable Systems. Cambridge University Press, Cambridge (1992) 
41. Bouten, L., van Handel, R.: On the separation principle of quantum control. In: Belavkin, V.P., Guta, M. (eds.) Quantum Stochastics and Information: Statistics, Filtering and Control (University of Nottingham, UK, 15-22 July 2006), pp. 206-238. World Scientific, Singapore (2008)

42. Szigeti, S.S., Carvalho, A.R.R., Morley, J.G., Hush, M.R.: Ignorance is bliss: general and robust cancellation of decoherence via no-knowledge quantum feedback. Phys. Rev. Lett. 113, 020407 (2014)

43. Milburn, G.J., Woolley, M.J.: An introduction to quantum optomechanics. Acta Phys. Slovaca 61(5), 483-601 (2011)

44. Poggio, M., Degen, C.L., Mamin, H.J., Rugar, D.: Feedback cooling of a cantilever's fundamental mode below 5 mK. Phys. Rev. Lett. 99, 017201 (2007)

45. Didier, N., Kamal, A., Oliver, W.D., Blais, A., Clerk, A.A.: Heisenberg-limited qubit read-out with two-mode squeezed light. Phys. Rev. Lett. 115, 093604 (2015)

46. Vidal, G., Werner, R.F.: Computable measure of entanglement. Phys. Rev. A 65, 032314 (2002)

47. Plenio, M.B.: The logarithmic negativity: A full entanglement monotone that is not convex. Phys. Rev. Lett. 95, 090503 (2005) 